\title{
Multiscale Real-Space Quantum-Mechanical Tight-Binding Calculations of Electronic Structure in Crystals with Defects using Perfectly Matched Layers
}

\author{
Hossein Pourmatin*, Kaushik Dayal ${ }^{\dagger}$ \\ Carnegie Mellon University
}

June 7, 2016

\begin{abstract}
We consider the scattering of incident plane-wave electrons from a defect in a crystal modeled by the time-harmonic Schrodinger equation. While the defect potential is localized, the far-field potential is periodic, unlike standard free-space scattering problems. Previous work on the Schrodinger equation has been almost entirely in free-space conditions; a few works on crystals have been in onedimension. We construct absorbing boundary conditions for this problem using perfectly matched layers in a tight-binding formulation. Using the example of a point defect in graphene, we examine the efficiency and convergence of the proposed absorbing boundary condition.
\end{abstract}

Keywords: Electron Scattering; Crystal Defects; Tight-binding; Waves in Periodic Media; Nonreflecting Boundary Conditions

\section{Introduction}

Electrons moving in perfect crystals are readily modeled through the periodic Schrodinger wave equation, and solutions are given by the classical Bloch / Floquet approach [AM76]. However, when the crystal has defects, the electronic wave scatters at these defects in complex ways. While electron scattering due to defects has been the focus of numerous theoretical and computational studies, many of these examine free-space scattering, e.g. [Sha12]; i.e., there is an isolated defect potential, and the electron feels no potential as it moves away to infinity. In contrast, defects in crystals are a perturbation of a periodic potential, and therefore the electron continues to feel a periodic potential even as it moves far away from the defect. In addition, scattering problems are most realistically posed on unbounded domains. Therefore, both analytical solutions and numerical methods are challenging for electron scattering at defects in crystals. However, this setting is most relevant to numerous current technological challenges, such as the electrical device properties of nanostructures, where defect scattering is the dominant mechanism of loss

*Email: mpourmat @andrew . cmu . edu

${ }^{\dagger}$ Email: kaushik@cmu.edu 
and resistance [Dat05]. Our focus in this paper is to develop a non-reflecting boundary condition that is suited to calculate the scattering of electrons at defects in otherwise-periodic crystals.

We work within the tight-binding (TB) model of quantum mechanics; this accounts for electron-electron interactions but in a mean-field sense. The Schrodinger equation for an $N$ electron system is posed in a $3 N$ dimensional space, therefore putting even extremely small molecules beyond current computing resources, let alone the possibility of examining defect scattering in large systems. For ground state calculations, empirical reductions of quantum mechanics to density functional theory (DFT) models are unable to capture key phenomena of interest in these materials; in particular, DFT has important qualitative failings in computing bandgaps and van der Waals interactions [KM12]. Given that bandstructure and bandgaps are the key quantities of interest in electronics applications, tight-binding has emerged as a popular model for such calculations [Dat05]. Tight-binding combines quantitative accuracy for bandgaps with first-principles electronic structure aspects - in particular, the atomic orbitals that are typically obtained from DFT - at a modest computational expense, albeit at the expense of a mean-field like approximation for electron-electron interactions through the effective potential felt by the electron. In any case, tight-binding is a widely-used physical model for electrons in crystals, and therefore we work with this well-characterized model.

Our strategy builds on ideas of non-reflecting boundary conditions that have been widely used in engineering and science in problems that are posed on unbounded domains. Non-reflecting boundary conditions and the closely-related Dirichlet-to-Neumann maps have been developed in a variety of settings: elastodynamics and geophysics, e.g. [BC03, $\left.\mathrm{BBG}^{+} 12\right]$; semiconductors, e.g. [Bas88]; electrostatics and electromagnetism, e.g. [MCCM96, CW94, DB07, YD11]; are a few examples. Broadly speaking, the idea behind absorbing boundary conditions is that they may be represented exactly through a nonlocal (integral) form that is expensive to evaluate, or through a local approximation [Giv91, Giv04].

In the specific context of quantum mechanics, the issue of unbounded domains has been addressed in various studies. For instance, a number of older works [Pan78, CS80, PBPL78, BP78, WFL82] have used Greens function approaches to solve the Schrodinger equation in a perturbed medium. Although very accurate, this approach has been limited to free-space scattering. In [LOvAK04], in addition to using supercells, they also examine raising the energy of atoms and bonds on the boundary of the computational domain. While physically motivated, it is difficult to systematically use this procedure in systems that are not already well-characterized. Recent important advances were made by Ehrhardt and Zheng [EZ08, ESZ09, Zhe08] in developing perfectly matched layers and absorbing boundary conditions. While they consider the case of systems where the potential is periodic far from the defect, they are confined to one dimension. Another contribution along these lines of absorbing boundary conditions, also in one dimension, is [ABK09].

A closely-related technique, that we use in this paper, is the idea of perfectly-matched layers (PML). The essential idea is to use dissipation in regions on the boundary of the computational truncated domain to absorb the outgoing wave. Generically, however, this causes spurious reflection. Perfectly-matched layers are constructed such that the damping is introduced in such a way as to thoretically have no spurious reflections; when discretized, however, some numerical error is introduced. An important advantage for our setting is that the periodicity outside the truncated domain can be readily handled. Perfectly-matched layers have been widely applied in a number of settings. For instance, [Ber94] showed that such layers can be constructed for Maxwell's equation in a way that they absorb all incoming energy without creating any reflecting wave. In [CW94], it was shown that these absorbing layers can be simply defined by complex coordinate stretching in three dimensions. It should be noted, however, that perfectly-matched layers were originally developed for homogeneous problems, and the property of perfect matching does 
not translate to inhomogeneous problems exactly [LLAT06]. However, perfectly-matched layers can nevertheless be used as an absorbing boundary condition:[OZAJ08] showed that by choosing a smooth absorption function, one can control the level of spurious reflection by appropriately increasing the width of the layer. In this work, we apply the perfectly matched layer method in inhomogeneous media, and find that indeed the results converge as the thickness of the absorbing layer increases. Further, we find that our formulation gives us faster convergence as the wavenumber increases.

\subsection{Organization}

The paper is organized as follows.

- In Section 2, we outline the tight-binding Schrodinger equation and transform the equations to a form amenable to applying the perfectly-matched layers method. We also describe the key details of the application of the model to graphene which is serves as our model system for demonstration.

- In Section 3, we describe the perfectly matched layer scheme.

- In Section 4, we present the results of our method.

\section{Notation.}

Boldface denotes vectors and tensors. We have used Einstein convention, i.e. repeated indices imply summation over those indices, except when noted.

$V(\boldsymbol{x})=V_{p}(\boldsymbol{x})+V_{d}(\boldsymbol{x})$ refer to the total potential, the periodic potential, and the defect potential respectively. $\phi(\boldsymbol{x})=\phi_{\boldsymbol{k}}(\boldsymbol{x})+\psi(\boldsymbol{x})$ refer to the total wavefunction, the incident Bloch wave function, and the scattered wavefunction respectively.

\section{Model Formulation}

\subsection{Schrodinger Equation and Decomposition into Incident and Scattered Prob- lems}

Our starting point is the time-harmonic (or steady-state) Schrodinger equation:

$$
-\frac{1}{2} \nabla^{2} \phi(\boldsymbol{x})+V(\boldsymbol{x}) \phi(\boldsymbol{x})=E \phi(\boldsymbol{x})
$$

In this eigenvalue problem, the eigenvalue $E$ is the energy of wavefunction $\phi(\boldsymbol{x})$.

Considering an almost-periodic medium with a defect, one can decompose the total potential $V(\boldsymbol{x})$ into a periodic potential $V_{p}(\boldsymbol{x})$ and a defect potential $V_{d}(\boldsymbol{x})$ :

$$
V(\boldsymbol{x})=V_{p}(\boldsymbol{x})+V_{d}(\boldsymbol{x})
$$

We assume that the defect potential, $V_{d}(\boldsymbol{x})$, has compact support and represents the defect or impurity in crystal. 
We next decompose the electronic wave function $\phi(\boldsymbol{x})$ into the incident Bloch plane wave $\phi_{\boldsymbol{k}}(\boldsymbol{x})$ and the scattered wave $\psi(\boldsymbol{x})$ :

$$
\phi(\boldsymbol{x})=\phi_{\boldsymbol{k}}(\boldsymbol{x})+\psi(\boldsymbol{x})
$$

Substituting these definitions lets us rewrite (2.1) as:

$$
-\frac{1}{2} \nabla^{2}\left(\phi_{\boldsymbol{k}}(\boldsymbol{x})+\psi(\boldsymbol{x})\right)+\left(V_{p}(\boldsymbol{x})+V_{d}(\boldsymbol{x})\right)\left(\phi_{\boldsymbol{k}}(\boldsymbol{x})+\psi(\boldsymbol{x})\right)=E\left(\phi_{\boldsymbol{k}}(\boldsymbol{x})+\psi(\boldsymbol{x})\right)
$$

Then, using that the incident Bloch plane wave $\phi_{\boldsymbol{k}}(\boldsymbol{x})$ is the solution to the Schrodinger equation in the perfectly-periodic medium, we can write:

$$
-\frac{1}{2} \nabla^{2} \phi_{\boldsymbol{k}}(\boldsymbol{x})+V_{p}(\boldsymbol{x}) \phi_{\boldsymbol{k}}(\boldsymbol{x})=E_{\boldsymbol{k}} \phi_{\boldsymbol{k}}(\boldsymbol{x})
$$

where $E_{\boldsymbol{k}}$ is the energy eigenvalue corresponding to an incident Bloch wave with wave number $\boldsymbol{k}$.

Subtracting (2.5) from (2.4), we get

$$
-\frac{1}{2} \nabla^{2} \psi(\boldsymbol{x})+\left(V_{p}(\boldsymbol{x})+V_{d}(\boldsymbol{x})\right) \psi(\boldsymbol{x})=E \psi(\boldsymbol{x})-V_{d}(\boldsymbol{x}) \phi_{\boldsymbol{k}}(\boldsymbol{x})+\left(E-E_{\boldsymbol{k}}\right) \phi_{\boldsymbol{k}}
$$

It is important to notice that even though the scattered wavefunction $\psi(\boldsymbol{x})$ is the direct result of the defect potential $V_{d}(\boldsymbol{x})$, it is also significantly influenced by the periodic potential $V_{p}(\boldsymbol{x})$. As a result, it is a scattering problem in an unbounded, inhomogeneous medium, and truncating the domain requires an absorbing boundary condition that accounts for this so as not to produce significant spurious reflections.

We now examine (2.6) in the limit $|\boldsymbol{x}| \rightarrow \infty$. The scattered wave $\psi(\boldsymbol{x})$, and all its derivatives, decay as $|\boldsymbol{x}| \rightarrow \infty$. In addition, $V_{p}(\boldsymbol{x})$ remains bounded as $|\boldsymbol{x}| \rightarrow \infty$, and $V_{d}(\boldsymbol{x})$ has local support. The only remaining term is $\left(E-E_{\boldsymbol{k}}\right) \phi_{\boldsymbol{k}}$, consequently we have:

$$
E=E_{k}
$$

Physically, this corresponds to elastic scattering: the energy of the scattered wave and the incident wave is the same, in all directions of scattering.

Using that $E=E_{k},(2.6)$ can be simplified to read:

$$
-\frac{1}{2} \nabla^{2} \psi(\boldsymbol{x})+\left(V_{p}(\boldsymbol{x})+V_{d}(\boldsymbol{x})\right) \psi(\boldsymbol{x})=E_{\boldsymbol{k}} \psi(\boldsymbol{x})-V_{d}(\boldsymbol{x}) \phi_{\boldsymbol{k}}(\boldsymbol{x})
$$

Now consider a truncated domain $\Omega_{c}$, which contains the support of potential $V_{d}(\boldsymbol{x})$, Fig. 1. We can write the weak-form of (2.8), using as the test function the complex conjugate of $\psi$. Note that $\psi$ vanishes at infinity.

$$
\begin{array}{r}
\int_{\Omega_{c}}\left[\frac{1}{2} \nabla \psi^{*} \cdot \nabla \psi+\psi^{*}(\boldsymbol{x})\left(V_{p}(\boldsymbol{x})+V_{d}(\boldsymbol{x})-E_{\boldsymbol{k}}\right) \psi(\boldsymbol{x})\right] \\
d \Omega_{c}-\int_{\Gamma_{c}} \frac{1}{2} \psi^{*}(\boldsymbol{x}) \nabla \psi(\boldsymbol{x}) \cdot \boldsymbol{n} d \Gamma_{c} \\
=-\int_{\Omega_{c}} \psi^{*}(\boldsymbol{x}) V_{d}(\boldsymbol{x}) \phi_{\boldsymbol{k}}(\boldsymbol{x}) d \Omega_{c}
\end{array}
$$

Our goal is to find the correct boundary condition to replace the surface integral in (2.9). Before turning to that in Section 3, we first describe the tight-binding approximation. 


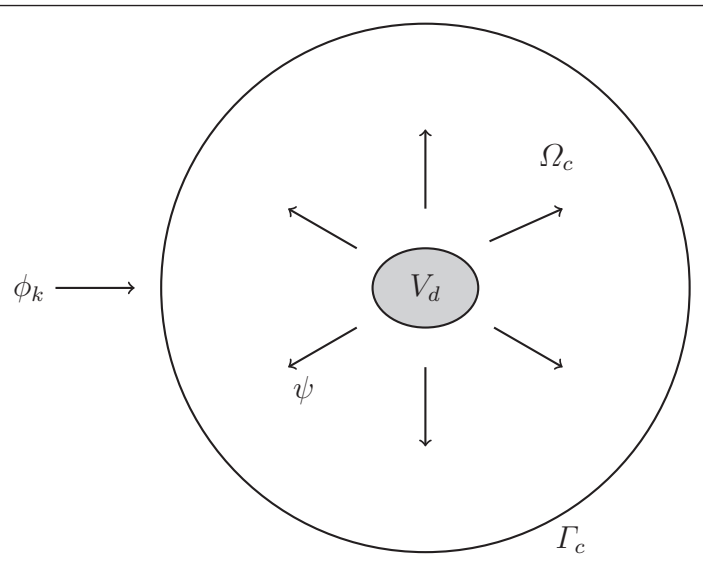

Figure 1: The incident wave, $\phi_{\boldsymbol{k}}$, and the scattered wave, $\psi$, in the truncated computational domain $\Omega_{c}$ with boundary $\Gamma_{c}$.

\subsection{Tight Binding Method}

The essential idea in the Tight-Binding method starts from the Linear Combination of Atomic Orbitals (LCAO) assumption to represent the scattered wavefunction $\psi(\boldsymbol{x})$ [BP78, Esc88]:

$$
\psi(\boldsymbol{x})=\sum_{j} \sum_{\alpha} a_{j \alpha} \Phi_{\alpha}\left(\boldsymbol{x}-\boldsymbol{X}_{j}\right)
$$

Here, $\Phi_{\alpha}\left(\boldsymbol{x}-\boldsymbol{X}_{j}\right)$ is a set of localized basis functions that represent the atomic orbitals. The $j$-th lattice site is located at $\boldsymbol{X}_{j}$, and $\alpha$ numbers the orbitals. In this paper, we have used self-consistent molecularorbitals for carbon [WJH69].

For a periodic system, however, the LCAO expression must satisfy the Bloch theorem [Mar08]. This states that the wavefunction in a periodic environment such as a crystal must have the form:

$$
\phi_{\boldsymbol{k}}(\boldsymbol{x})=e^{i \boldsymbol{k} \cdot \boldsymbol{x}} u_{\boldsymbol{k}}(\boldsymbol{x})
$$

where $\boldsymbol{k}$ is the wave number, and $u_{\boldsymbol{k}}(\boldsymbol{x})$ is a periodic function.

This form can be written in terms of the LCAO through the following summation over localized orbitals (known as the Bloch Sum):

$$
\phi_{\boldsymbol{k}}(\boldsymbol{x})=\sum_{j} \sum_{\alpha} c_{\alpha} e^{i \boldsymbol{k} \cdot \boldsymbol{X}_{j}} \Phi_{\alpha}\left(\boldsymbol{x}-\boldsymbol{X}_{j}\right)
$$

The first sum is over unit cells, the $i$-th of which is specified by the Bravais lattice vector $\boldsymbol{X}_{i}$. The second sum is over the set of orbitals $\alpha$ which are assumed to be centered on each site and participating in the bonding. For graphene, there are two atoms per unit cell (Fig. 3), and we therefore require a further sum over the atoms within the unit cell, but this is not shown explicitly for brevity.

\subsection{Implementation of Tight-Binding for Graphene}

We consider graphene as a model system to demonstrate our method. A single graphene sheet consists of carbon atoms in a two-dimensional hexagonal lattice. Fig. 2 shows that the graphene sheet can be 
generated from a unit cell consisting of 2 atoms by the lattice vectors $\boldsymbol{a}_{1}$ and $\boldsymbol{a}_{2}$ :

$$
\boldsymbol{a}_{1}=\frac{\sqrt{3} a}{2} \boldsymbol{e}_{1}+\frac{a}{2} \boldsymbol{e}_{2}, \quad \boldsymbol{a}_{2}=\frac{\sqrt{3} a}{2} \boldsymbol{e}_{1}-\frac{a}{2} \boldsymbol{e}_{2}
$$

The position of each carbon atom in the graphene sheet is given by starting at one of the two atoms in the unit cell and adding an integer linear combination of $\boldsymbol{a}_{1}$ and $\boldsymbol{a}_{2}$ :

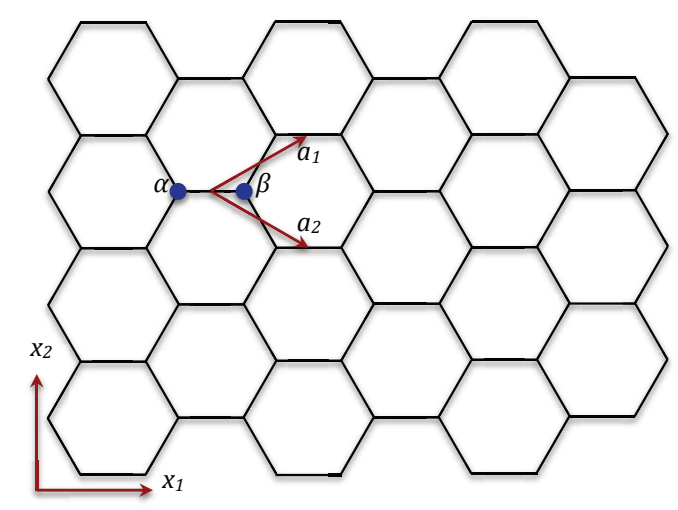

Figure 2: Schematic of a graphene sheet showing the lattice vectors.

Knowing the lattice vectors $a_{1}$ and $a_{2}$ and the positions of the two carbon atoms labeled by 1 and $2, X_{n}$, we can construct the whole $2 D$ Graphene layer as [AD12]:

$$
\boldsymbol{X}_{n,\left(\zeta_{1}, \zeta_{2}\right)}=\zeta_{1} \boldsymbol{a}_{1}+\zeta_{2} \boldsymbol{a}_{2}+\boldsymbol{X}_{n}, \quad n=1,2
$$

In the Tight-Binding method one assumes that the valence electrons are "tightly bound" to the atoms, and that the electronic orbitals are not strongly altered during the bonding process [TM11, ZJD09].

\subsection{The Hamiltonian and Overlap Matrices}

Consider the incident Bloch wave given by (2.5). Using (2.12), the eigenvalue problem corresponding to each wave number $\boldsymbol{k}$ is given by

$$
\sum_{j} h_{\alpha \beta}^{j} e^{i \boldsymbol{k} \cdot \boldsymbol{X}_{j}} c_{k \beta}=E_{\boldsymbol{k}} \sum_{j} s_{\alpha \beta}^{j} e^{i \boldsymbol{k} \cdot \boldsymbol{X}_{j}} c_{k \beta}
$$

where $E_{k}$ is the eigenvalue. The quantities $h_{\alpha \beta}$ and $s_{\alpha \beta}$ are the components of the Hamiltonian and Overlap matrices, respectively:

$$
\begin{aligned}
h_{\alpha \beta}^{j} & =\int_{\Omega}\left[-\frac{1}{2} \nabla^{2} \Phi_{\alpha}^{*}\left(\boldsymbol{x}-\boldsymbol{X}_{0}\right) \Phi_{\beta}\left(\boldsymbol{x}-\boldsymbol{X}_{j}\right)+\Phi_{\alpha}^{*}\left(\boldsymbol{x}-\boldsymbol{X}_{0}\right) V_{p}(\boldsymbol{x}) \Phi_{\beta}\left(\boldsymbol{x}-\boldsymbol{X}_{j}\right)\right] d \Omega \\
s_{\alpha \beta}^{j} & =\int_{\Omega} \Phi_{\alpha}^{*}\left(\boldsymbol{x}-\boldsymbol{X}_{0}\right) \Phi_{\beta}\left(\boldsymbol{x}-\boldsymbol{X}_{j}\right) d \Omega
\end{aligned}
$$




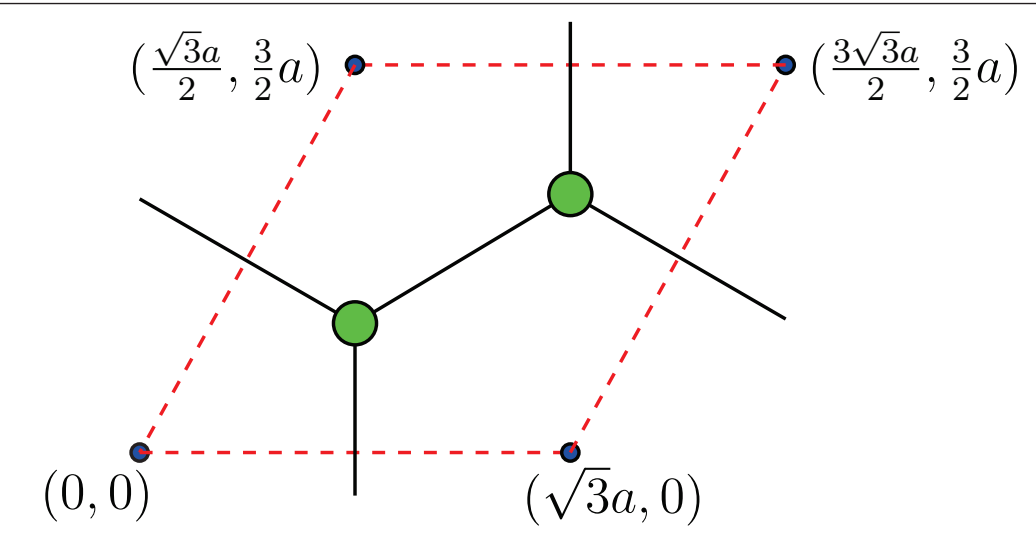

Figure 3: A unit cell of graphene with Lattice constant $\sqrt{3} a$, and $a=1.42 \AA$. Each unit cell contains two atoms shown in green.

If $V_{p}$ is a given function, the integrals above are called two-center integrals when $\alpha \neq \beta$, and one-center or on-site integrals when $\alpha=\beta$ [TM11]. However, typically $V_{p}(\boldsymbol{x})$ is a sum of atom-centered potentials $V_{\gamma}\left(\boldsymbol{x}-\boldsymbol{X}_{\gamma}\right)$, each of which is independent of all the other atoms in the system:

$$
V_{p}(\boldsymbol{x})=\sum_{\gamma} V_{\gamma}\left(\boldsymbol{x}-\boldsymbol{X}_{\gamma}\right)
$$

This assumption introduces the three-center integrals for the second term in $h_{\alpha \beta}^{i}$, where $\alpha, \beta$ and $\gamma$ are different. While the one- and two-center terms are relatively straightforward, the three-center terms are much more challenging. Standard practice in tight-binding is to set these to zero identically [TM11]. In this paper, the periodic potential, $V_{p}$ is calculated based on [May04].

It is standard practice to only consider the 3 nearest neighbors of an atom when computing the Hamiltonian and Overlap matrices. This is usually justified based on the fact that the orbitals $\Phi\left(\boldsymbol{x}-\boldsymbol{X}_{i}\right)$ are highly localized. However, we have considered 12 nearest neighbors as show in Fig. 4. Although this seems to make the calculations very expensive compared to the usual 3 nearest neighbor assumption, note that one needs to perform these integrations only once. As shown in Fig. 5 for the convergence of $h_{11}$, the error is significantly reduced by considering the larger set of neighbors.

As a test of the tight-binding approximation scheme, the dispersion relation between $\boldsymbol{k}$ and the energy $E$ in graphene is plotted for the first Brillouin zone in Fig. 6. We recover the important features such as the Dirac cones at the K-points.

\section{Perfectly Matched Layers for the Schrodinger Equation}

In this paper, we adopt the complex coordinate stretching approach, originally developed by [CW94]. For this method, we introduce a narrow absorbing layer in the vicinity of the truncated computational boundary $\Gamma_{c}$, as shown in Fig. 7. $\Gamma_{a}$ is the interface between the propagating domain and the absorbing layer. 


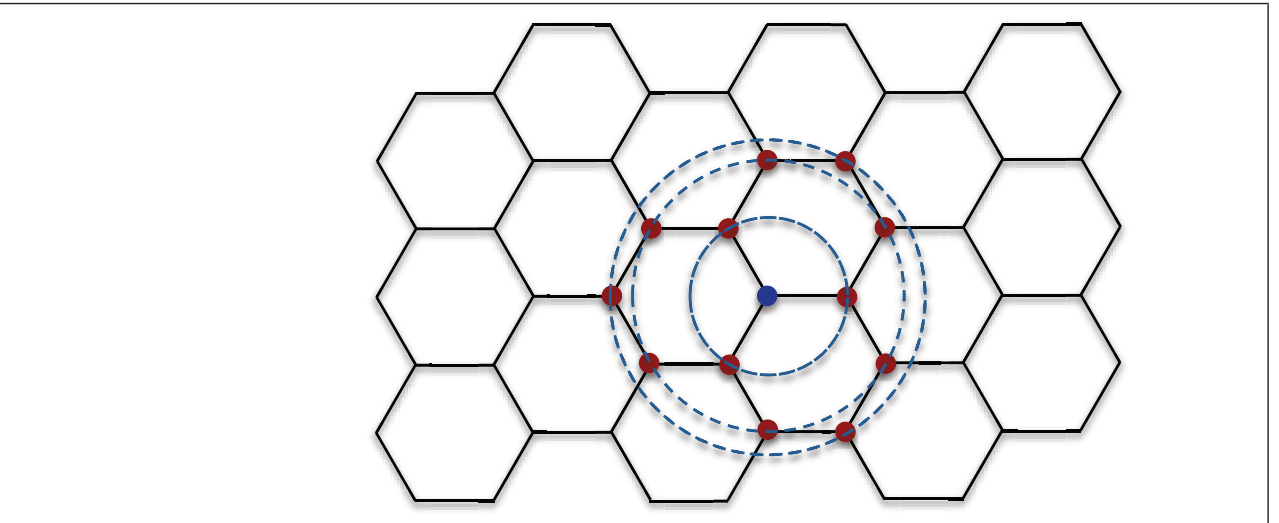

Figure 4: The 12 nearest neighbors for the blue atom in a graphene sheet.

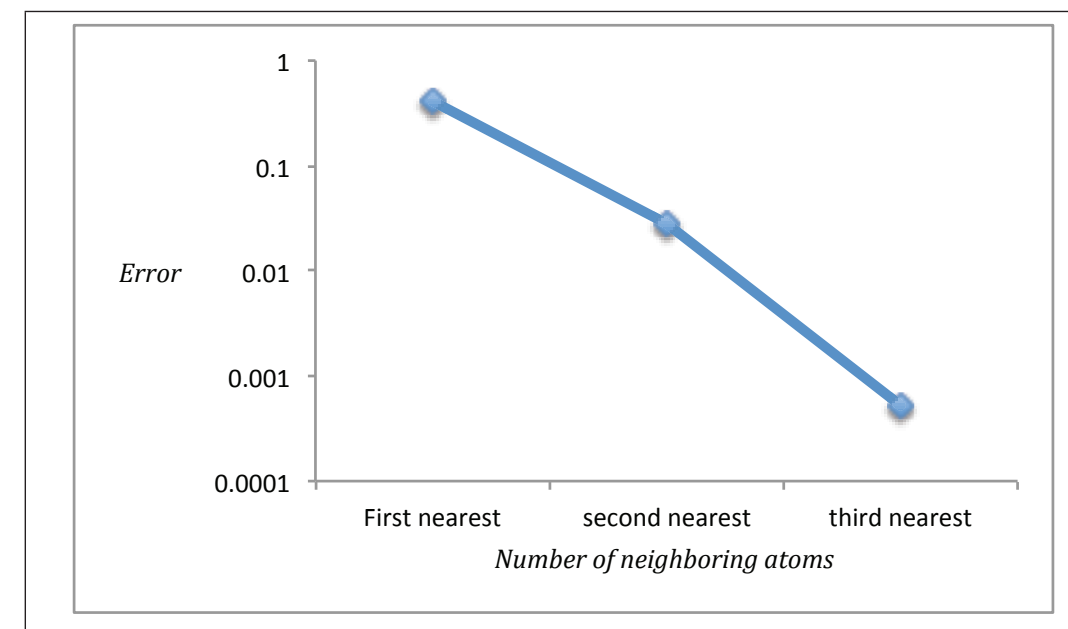

Figure 5: Convergence of $h_{11}$ as more neighboring atoms are accounted for.

The basic idea of perfectly-matched layers is to absorb the energy of the traveling waves without causing any reflection, so that when the wave reaches the boundary of the truncated domain, its reflection will be insignificant. As a result, one can simply apply a zero Dirichlet or Neumann boundary condition on $\Gamma_{c}$. However, as noted by [OZAJ08] and others, the Perfectly Matched Layer method fails to be truly reflectionless in inhomogeneous media. We will show that one can reduce the reflection, introduced by the discretization and the periodicity of the medium, by increasing the thickness of the absorbing layer.

The absorbing layer can be constructed by stretching the position vector into a complex plane [CW94]. This will cause the traveling wave to decay exponentially. However, since we are fictitiously changing the distance between the atoms, the potential energy will change as well. As a result, artificial reflection between domains is inevitable; however, one can reduce this reflection to a negligible level through a smooth stretching. Therefore, we define the complex stretching in the following way:

$$
\tilde{\boldsymbol{x}}=\boldsymbol{x}+\imath \boldsymbol{\sigma}(\boldsymbol{x})
$$

where $\sigma$ is the absorption vector function that acts as a damping coefficient. When chosen this way, the 


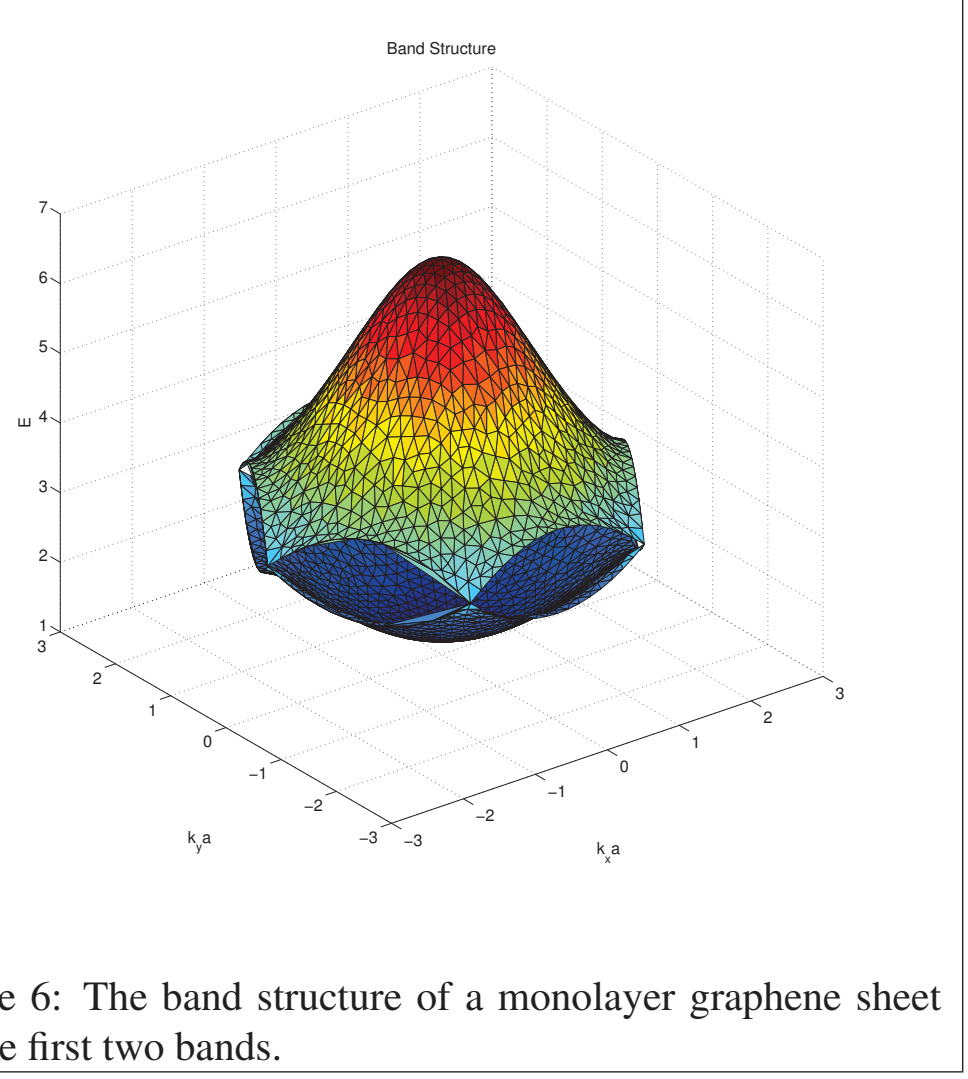

Figure 6: The band structure of a monolayer graphene sheet for the first two bands.

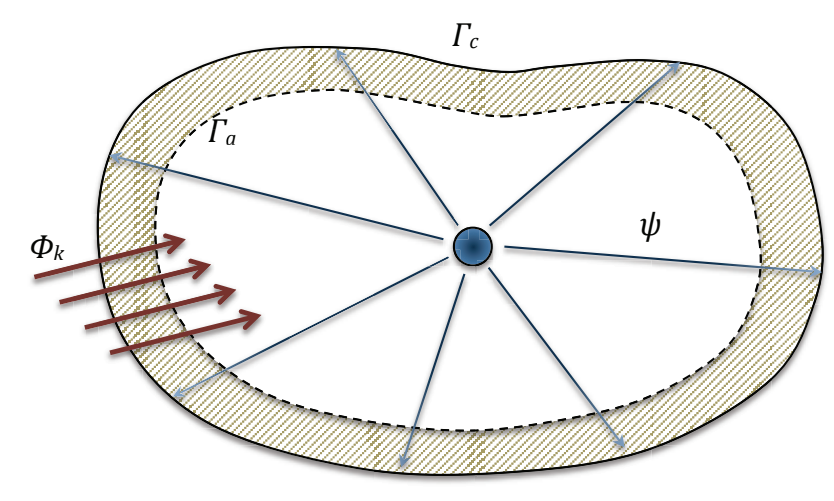

Figure 7: Schematic absorbing layer around the boundary $\Gamma_{c}$.

damping will act on radial waves to attenuate them exponentially, while it will have no effect on waves that travel parallel to the boundary. This feature is a key difference between the perfectly-matched layer method and the adiabatic methods, which cause waves propagating in any direction to decay.

In order to maintain the original form of the equation inside the domain, and avoid significant spurious reflections, the absorption function must be zero on the propagation domain. Following [BP06, KJP09], we define $\boldsymbol{\sigma}$ as a smooth function varying from $\boldsymbol{0}$ at some point $\boldsymbol{x}_{0}$ on $\Gamma_{a}$, to $\sigma_{0}$ at $\boldsymbol{x}_{1}$ on $\Gamma_{c}$. [OZAJ08] showed that the smoother the absorption function, the faster the solution converges as the thickness of 
the absorbing layer increases. We define the absorption as the real function:

$$
\boldsymbol{\sigma}(x)=\sigma_{0} \frac{\int_{\boldsymbol{x}_{0}}^{\boldsymbol{x}}\left|\left(\boldsymbol{t}-\boldsymbol{x}_{0}\right)\right|^{2}\left|\left(\boldsymbol{x}_{1}-\boldsymbol{t}\right)\right|^{2} d \boldsymbol{t}}{\left.\left|\int_{\boldsymbol{x}_{0}}^{\boldsymbol{x}_{1}}\right|\left(\boldsymbol{t}-\boldsymbol{x}_{0}\right)\right|^{2}\left|\left(\boldsymbol{x}_{1}-\boldsymbol{t}\right)\right|^{2} d \boldsymbol{t} \mid}
$$

Note that the increase of the width of the absorbing layer makes the transition from the original domain to the absorbing layer smoother, thereby causing less reflection. As mentioned earlier, the exponential attenuation, caused by the absorbing layer, makes the amplitude of the scattering wavefunction negligible. So one can now apply a zero Dirichlet or Neumann boundary condition on boundary $\Gamma_{c}$, without causing any extra reflection. After applying zero Neumann boundary condition, the weak-form in (2.9) will be:

$$
\begin{aligned}
& \int_{\Omega_{c}}\left(\psi_{, m}^{*} F_{m p}^{-1} \psi_{, n} F_{n p}^{-1}+\psi^{*}(\boldsymbol{x})\left(V_{p}(\boldsymbol{x})+V_{d}(\boldsymbol{x})-E_{k}\right) \psi(\boldsymbol{x})\right) J d v \\
& =-\int_{\Omega_{c}} \psi^{*}(\boldsymbol{x}) V_{d}(\boldsymbol{x}) \phi_{k}(\boldsymbol{x}) J d v
\end{aligned}
$$

where $F_{i j}=\frac{\partial \tilde{x}_{i}}{\partial x_{j}}$ and $J=\operatorname{det}(\boldsymbol{F})$. Note that (3.3) requires that the truncated computational domain must contain the support of the defect potential $V_{d}$.

We emphasize an important difference between the perfectly-matched layer method vs. a regular damping method where one simply adds an imaginary potential to the Schrodinger equation as in [HIKH96]. As shown in (3.3), we gradually transform both the potential and kinetic terms to the complex plane. This is the physics behind the perfectly matched absorbing layers in free space. However, in the above-cited damping methods, only the potential term changes. Those methods will always cause reflection, even in homogeneous domains.

\section{Examples}

We implement the perfectly-matched layer methods on a monolayer graphene sheet with various types of defects. In particular, we look at a single vacancy, a di-vacancy, and a tri-vacancy, and examine the effect of the different defects on the scattering.

We subject each of these defect configurations to the same incident Bloch wave $\phi_{\boldsymbol{k}}(\boldsymbol{x})$ with wave-vector $\boldsymbol{k}=0.42 \boldsymbol{e}_{1}+0.49 \boldsymbol{e}_{2}$; this wave-vector is chosen as a typical representative. In these examples, the size of the computational domain is $12.9 \times 12.6 \mathrm{~nm}$ and the thickness of the absorbing layer is $1.5 \mathrm{~nm}$ in all directions. The defects at the center of the domain scatter the the Bloch wave $\phi_{\boldsymbol{k}}(\boldsymbol{x})$. We assume that each atom in the graphene sheet is rigidly held in place by external forces to avoid complications of inelastic scattering and momentum transfer to the graphene sheet.

Fig. 8 shows the electron density field $\rho(\boldsymbol{x})=\phi^{*}(\boldsymbol{x}) \phi(\boldsymbol{x})$ obtained by our method for all three defect configurations. Although the vacancies have been chosen to be symmetric with respect to the vertical axis, the density of the scattering wave is not symmetric. This is because of the strong influence of the incident wave on the scattering (2.9).. This is particularly pronounced in the case of the tri-vacancy. We notice that the scattering profiles for each of the defect configurations is very different, and hence defect configuration and density can be expected to have a significant effect on electronic conductivity, and is of relevance to conductivity and loss in graphene-based electronic devices. 
As expected from free-space calculations that have the same asymptotic scaling, the norm of the scattering wave $\psi^{*}(\boldsymbol{x}) \psi(\boldsymbol{x})$ shown in Fig. 9 decays as $1 / r$ away from the defect, until it reaches the absorbing layer. From that point it decays exponentially and completely vanishes before reaching the boundary of the computational domain. As a result, there are no spurious reflections from the Dirichlet boundary conditions that are applied on the boundaries of the computational domain.

In order to evaluate the convergence of the PML method for this example, we note that the central purpose of our absorbing boundary condition is to be transparent to the outgoing waves. Consequently, if our boundary condition was performing ideally, changing the truncated computational domain would not change the results, as long as the domain contains the support of the defect potential $V_{d}(\boldsymbol{x})$. This fact leads us to examine the convergence and error in the solution by computing the change in the solution when we translate the truncated domain; alternately, we could think of it as moving the defect within the domain, as shown in Fig. 10. We evaluate the error using the expression:

$$
E=\int \frac{\left|\rho(\boldsymbol{x})-\rho\left(\boldsymbol{x}^{\prime}\right)\right|^{2}}{\rho(\boldsymbol{x})^{2}}
$$

where the integration is over a region that is also translated.

Running the set of simulations for different layer thicknesses shows satisfactory convergence for the PML method. Fig. 11 shows this convergence for two different incident waves. In addition, we find that the accuracy of the method improves as the norm of the wavenumber increases; an example of this trend is shown in Fig. 11. This is to be expected as the method is based in part on the application of linear damping which has a bigger impact on higher spatial frequencies. This is in contrast to many existing non-reflecting boundary conditions that rapidly lose their accuracy as the wavenumber increases, e.g. [EM77].

\section{Conclusion}

The time-harmonic Schrodinger equation for an almost-periodic crystal with localized defects was considered. Perfectly-matched layers were considered as an absorbing boundary condition and investigated. We find that the method is reliable and can be used for periodic problems when the absorbing profile is sufficiently smooth and as the thickness of the absorbing layer increases. This suggests that the PML method can be used for any incident wave vector and energy in the periodic media.

While tight-binding is superior to density functional theory for important properties such as bandstructures, DFT is extremely useful for structural properties. Therefore, it is useful to extend the method that we have described to DFT. In the locally self-consistent multiple scattering (LSMS) formulation of DFT - an order $N$ method that can handle non-periodic systems with defects - the problem is posed in terms of electron scattering [WSS ${ }^{+}$55]. LSMS hence provides a natural framework for the use of the methods described here to appropriately truncate the domain without spurious effects. Extending our method to LSMS and related numerical methods for electronic structure [KM09, MK12] is an area of our current activity. 


\section{Acknowledgments}

We thank Jacobo Bielak and Mike Widom for useful discussions. For financial support, we thank NSF Mechanics of Materials (CAREER 1150002), AFOSR Computational Mathematics (YI FA9550-12-10350), ARO Numerical Analysis (YI W911NF-12-1-0156), and ONR Applied and Computational Analysis (N00014-14-1-0715). Kaushik Dayal also thanks Carnegie Mellon University College of Engineering for an Early Career Fellowship. This research was also supported in part by the National Science Foundation through TeraGrid resources provided by Pittsburgh Supercomputing Center.

\section{References}

[ABK09] Xavier Antoine, Christophe Besse, and Pauline Klein, Absorbing boundary conditions for the one-dimensional schr\&\#246; dinger equation with an exterior repulsive potential, J. Comput. Phys. 228 (2009), no. 2, 312-335.

[AD12] E Akatyeva and T Dumitrică, Chiral graphene nanoribbons: Objective molecular dynamics simulations and phase-transition modeling, The Journal of chemical physics 137 (2012), no. $23,234702$.

[AM76] Neil W Ashcroft and N David Mermin, Solid state phys, Saunders, Philadelphia (1976), 293.

[Bas88] G. Bastard, Wave mechanics applied to semiconductor heterostructures, Monographies de physique, Les Éditions de Physique, 1988.

$\left[\mathrm{BBG}^{+} 12\right]$ Daniel Baffet, Jacobo Bielak, Dan Givoli, Thomas Hagstrom, and Daniel Rabinovich, Long-time stable high-order absorbing boundary conditions for elastodynamics, Computer Methods in Applied Mechanics and Engineering 241-244 (2012), no. 0, 20 - 37.

[BC03] Ushnish Basu and Anil K. Chopra, Perfectly matched layers for time-harmonic elastodynamics of unbounded domains: theory and finite-element implementation, Computer Methods in Applied Mechanics and Engineering 192 (2003), no. 11-12, 1337 - 1375.

[Ber94] Jean-Pierre Berenger, A perfectly matched layer for the absorption of electromagnetic waves, Journal of Computational Physics 114 (1994), no. 2, 185 - 200.

[BP78] J. Bernholc and Sokrates T. Pantelides, Scattering-theoretic method for defects in semiconductors. $i$. tight-binding description of vacancies in si, ge, and gaas, Phys. Rev. B 18 (1978), 1780-1789.

[BP06] James H. Bramble and Joseph E. Pasciak, Analysis of a finite pml approximation for the three dimensional time-harmonic maxwell and acoustic scattering problems, MATH. COMP (2006), 597-614.

[CS80] Joseph Callaway and S. P. Singhal, Scattering theory of defects in solids: Theory and application to the atomic vacancy, International Journal of Quantum Chemistry 18 (1980), no. S14, 651-663. 
[CW94] Weng Cho Chew and William H. Weedon, A 3d perfectly matched medium from modified maxwell's equations with stretched coordinates, Microwave and Optical Technology Letters 7 (1994), no. 13, 599-604.

[Dat05] Supriyo Datta, Quantum transport: atom to transistor, Cambridge University Press, 2005.

[DB07] Kaushik Dayal and Kaushik Bhattacharya, A real-space non-local phase-field model of ferroelectric domain patterns in complex geometries, Acta materialia 55 (2007), no. 6, 19071917.

[EM77] BjËErn Engquist and Andrew Majda, Absorbing boundary conditions for numerical simulation of waves, Proceedings of the National Academy of Sciences 74 (1977), no. 5, 17651766.

[Esc88] H Eschrig, Optimized lcao method and the electronic structure of extended systems, Springer-Verlag, 1988.

[ESZ09] Matthias Ehrhardt, Jiguang Sun, and Chunxiong Zheng, Evaluation of scattering operators for semi-infinite periodic arrays, Communications in Mathematical Sciences 7 (2009), no. 2, 347-364.

[EZ08] Matthias Ehrhardt and Chunxiong Zheng, Exact artificial boundary conditions for problems with periodic structures, Journal of Computational Physics 227 (2008), no. 14, 6877 - 6894.

[Giv91] Dan Givoli, Non-reflecting boundary conditions, J. Comput. Phys. 94 (1991), no. 1, 1-29.

[Giv04] Dan Givoli, High-order local non-reflecting boundary conditions: a review, Wave Motion 39 (2004), no. 4, 319 - 326, <ce:title>New computational methods for wave propagation</ce:title $>$.

[HIKH96] Youhong Huang, Srinivasan S. Iyengar, Donald J. Kouri, and David K. Hoffman, Further analysis of solutions to the time-independent wave packet equations of quantum dynamics. ii. scattering as a continuous function of energy using finite, discrete approximate hamiltonians, Environmental and Ecological Statistics 105 (1996), no. 3, 927-939.

[KJP09] Seungil Kim, Joseph, and E. Pasciak, The computation of resonances in open systems using a perfectly matched layer, Math. Comp (2009).

[KM09] Raguraman Kannan and Arif Masud, Stabilized finite element methods for the schrödinger wave equation, Journal of Applied Mechanics 76 (2009), no. 2, 021203.

[KM12] Jiří Klimeš and Angelos Michaelides, Perspective: Advances and challenges in treating van der waals dispersion forces in density functional theory, The Journal of chemical physics 137 (2012), no. 12, 120901.

[LLAT06] Shaofan Li, Xiaohu Liu, Ashutosh Agrawal, and Albert C. To, Perfectly matched multiscale simulations for discrete lattice systems: Extension to multiple dimensions, Phys. Rev. B 74 (2006), 045418.

[LOvAK04] Seungwon Lee, Fabiano Oyafuso, Paul von Allmen, and Gerhard Klimeck, Boundary conditions for the electronic structure of finite-extent embedded semiconductor nanostructures, Physical Review B 69 (2004), no. 4, 045316. 
[Mar08] Richard M. Martin, Electronic structure: Basic theory and practical methods, Cambridge, 2008.

[May04] A. Mayer, Band structure and transport properties of carbon nanotubes using a local pseudopotential and a transfer-matrix technique, Carbon 42 (2004), no. 10, 2057 - 2066.

[MCCM96] Peter Monk, Francis Collino, Francis Collino, and Et Peter Monk, The perfectly matched layer in curvilinear coordinates, SIAM J. Sci. Comput 19 (1996), 2061-2090.

[MK12] Arif Masud and Raguraman Kannan, B-splines and nurbs based finite element methods for kohn-sham equations, Computer Methods in Applied Mechanics and Engineering 241 (2012), 112-127.

[OZAJ08] Ardavan F. Oskooi, Lei Zhang, Yehuda Avniel, and Steven G. Johnson, The failure of perfectly matched layers, and towards their redemptionby adiabatic absorbers, Opt. Express 16 (2008), no. 15, 11376-11392.

[Pan78] Sokrates T. Pantelides, The electronic structure of impurities and other point defects in semiconductors, Rev. Mod. Phys. 50 (1978), 797-858.

[PBPL78] S. T. Pantelides, J. Bernholc, J. Pollmann, and N. O. Lipari, Green's function scatteringtheoretic methods for point defects, surfaces, and interfaces in solids, International Journal of Quantum Chemistry 14 (1978), no. S12, 507-521.

[Sha12] Ramamurti Shankar, Principles of quantum mechanics, Springer Science \& Business Media, 2012.

[TM11] Ellad B Tadmor and Ronald E Miller, Modeling materials: continuum, atomistic and multiscale techniques, Cambridge University Press, 2011.

[WFL82] A. R. Williams, Peter J. Feibelman, and N. D. Lang, Green's-function methods for electronic-structure calculations, Phys. Rev. B 26 (1982), 5433-5444.

[WJH69] J. A. Pople W. J. Hehre, R. F. Stewart, Self-Consistent Molecular-Orbital Methods. I. Use of Gaussian Expansions of Slater-Type Atomic Orbitals, Journal of Chemical Physics (1969).

[WSS ${ }^{+95] ~ Y a n g ~ W a n g, ~ G M ~ S t o c k s, ~ W A ~ S h e l t o n, ~ D M C ~ N i c h o l s o n, ~ Z ~ S z o t e k, ~ a n d ~ W M ~ T e m m e r m a n, ~}$ Order-n multiple scattering approach to electronic structure calculations, Physical review letters 75 (1995), no. 15, 2867.

[YD11] Lun Yang and Kaushik Dayal, A completely iterative method for the infinite domain electrostatic problem with nonlinear dielectric media, Journal of Computational Physics 230 (2011), no. 21, 7821-7829.

[Zhe08] Chunxiong Zheng, An exact absorbing boundary condition for the schrödinger equation with sinusoidal potentials at infinity. commun, Comput. Phys 3 (2008), no. 3, 641-658.

[ZJD09] D-B Zhang, RD James, and T Dumitrică, Electromechanical characterization of carbon nanotubes in torsion via symmetry adapted tight-binding objective molecular dynamics, Physical Review B 80 (2009), no. 11, 115418. 

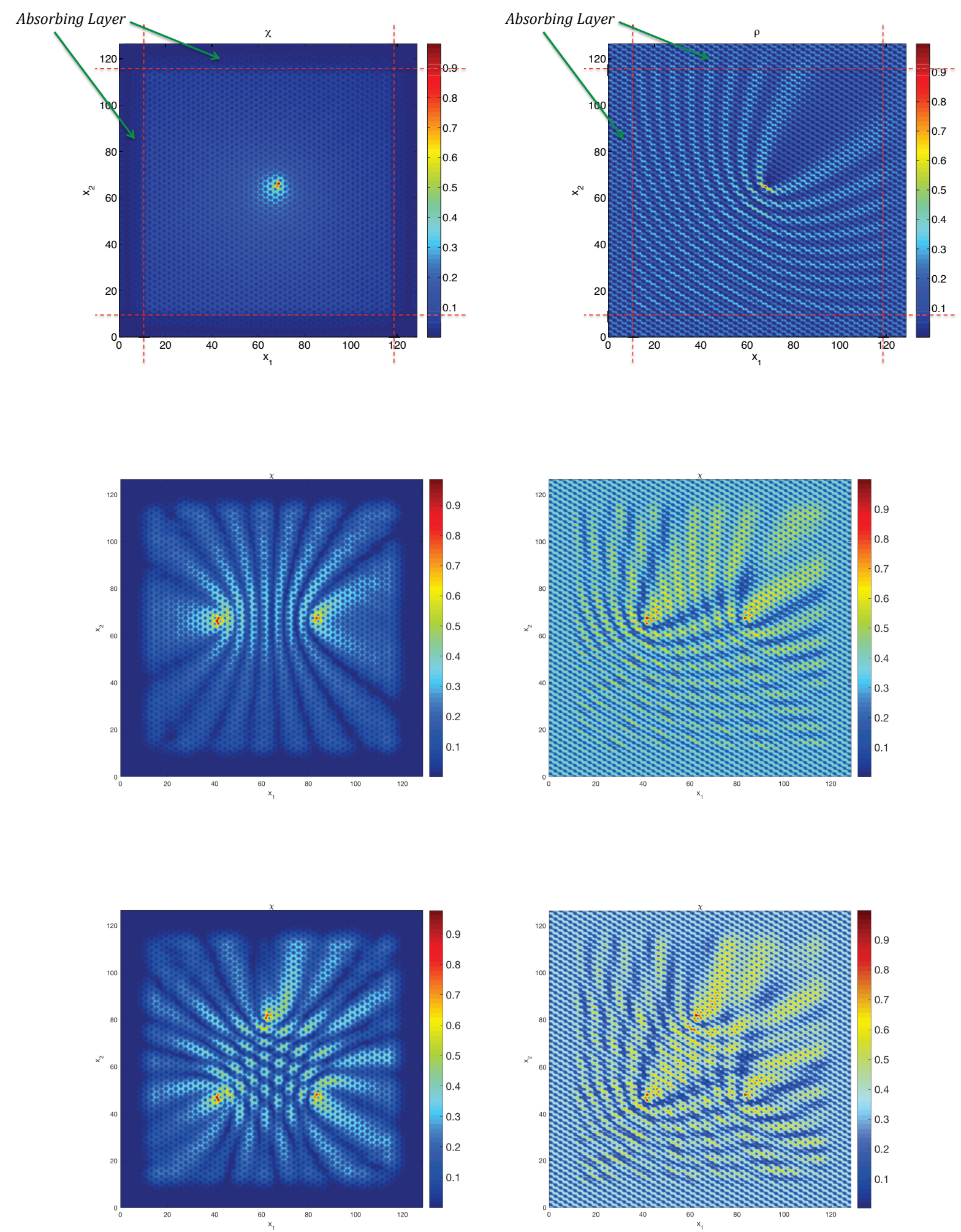

Figure 8: The electron density field in a graphene sheet around a point defect. Left column: the scattered wave; right column: the scattered and incident wave superposed. The top row is a single vacancy, the middle row is a di-vacancy, and the bottom row is a tri-vacancy. The incident wave has wave-vector $\boldsymbol{k}=0.42 \boldsymbol{e}_{1}+0.49 \boldsymbol{e}_{2}$. 


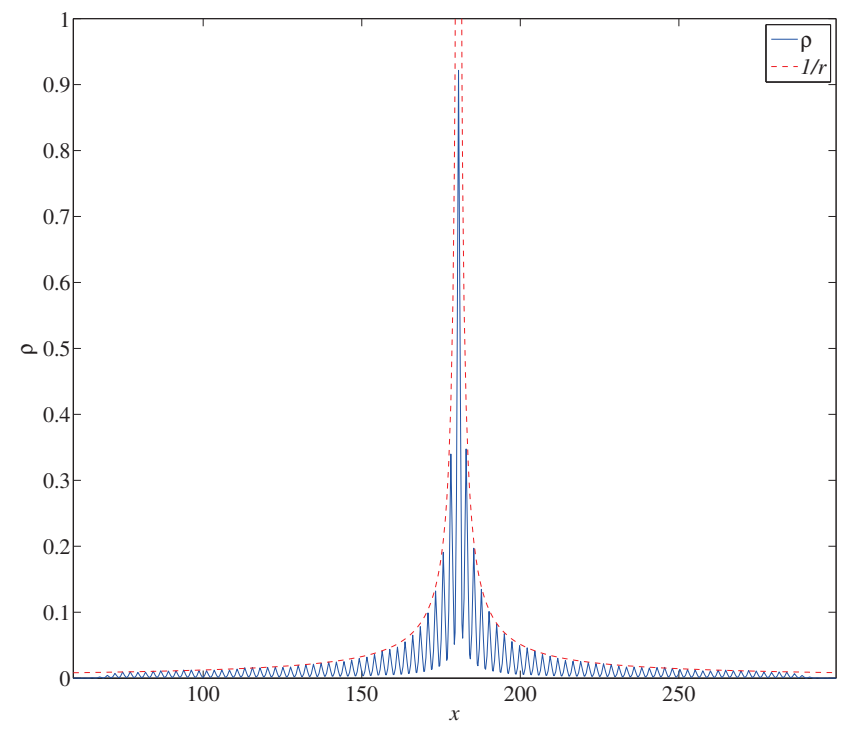

Figure 9: Profile of scattered wave density for a constant $x_{2}$, compared to $1 / r$ profile. The effect of the absorbing layer can be seen at the edges.

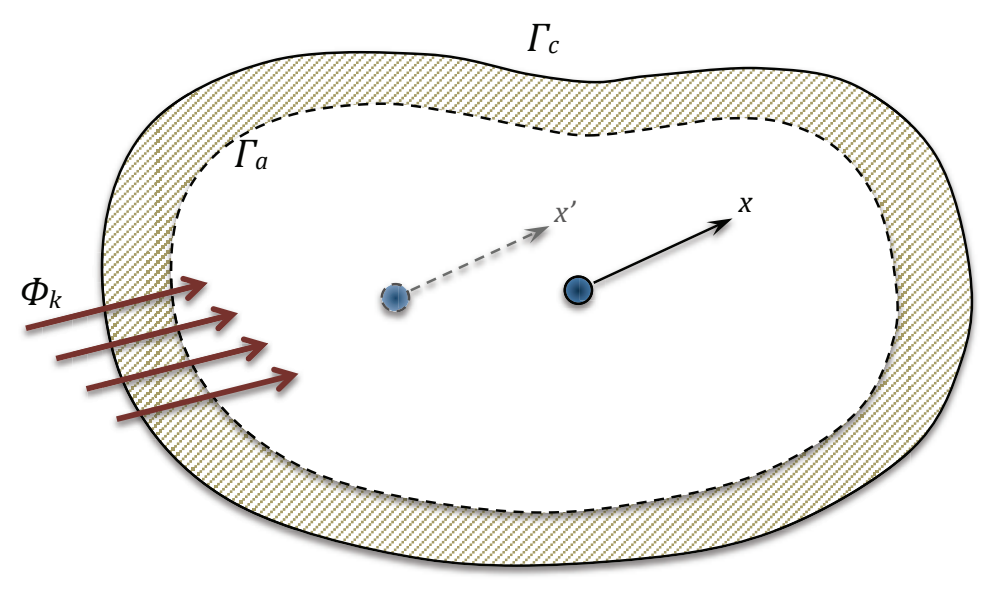

Figure 10: The electronic density $\rho$ at point $\boldsymbol{x}$ and $\boldsymbol{x}^{\prime}$ must be the same for the appropriate position of the defect. 


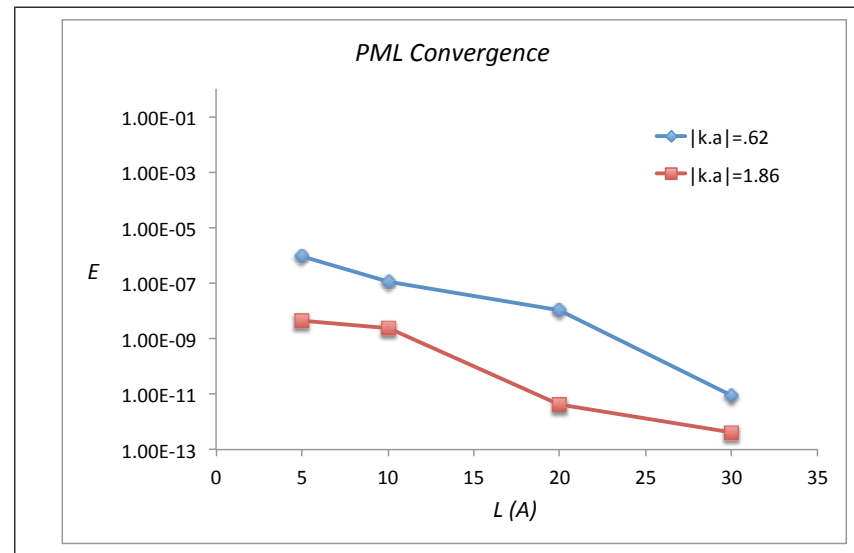

Figure 11: Plot of the error $E$ shows convergence of the solution as the thickness of the layer increases. 
Absorbing Layer

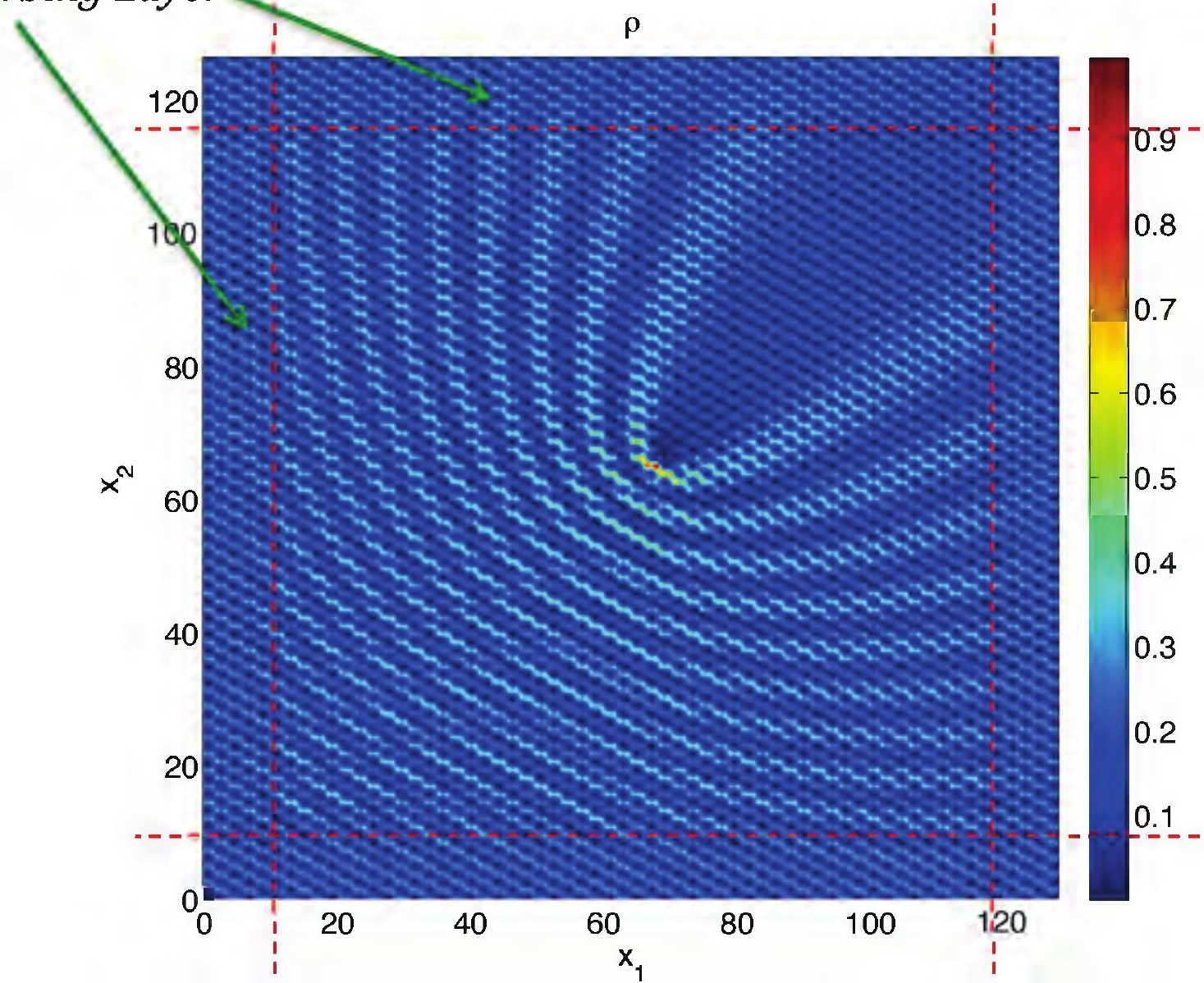

